\title{
Hybrid Capillary Discharge Waveguide for Laser Wakefield Acceleration
}

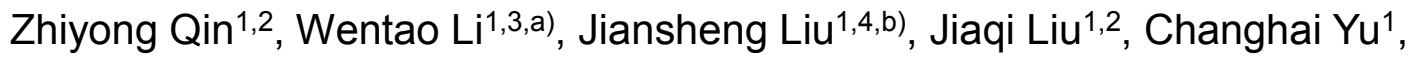 \\ Wentao Wang ${ }^{1}$, Rong Qi ${ }^{1}$, Zhijun Zhang ${ }^{1}$, Ming Fang ${ }^{1,2}$, Ke Feng ${ }^{1,2}$, Ying Wu ${ }^{1,2}$, \\ Lintong $\mathrm{Ke}^{1,2}$, Yu Chen ${ }^{1,2}$, Cheng Wang ${ }^{1}$, Ruxin $\mathrm{Li}^{1,4,5}$, and Zhizhan $\mathrm{Xu}^{1,4,5}$ \\ ${ }^{1}$ State Key Laboratory of High Field Laser Physics, Shanghai Institute of Optics and Fine Mechanics, Chinese Academy \\ of Sciences, Shanghai 201800, China \\ ${ }^{2}$ University of Chinese Academy of Sciences, Beijing 100049, China \\ ${ }^{3}$ Department of Physics, SUPA and University of Strathclyde, Glasgow G4 0NG, United Kingdom \\ ${ }^{4}$ Collaborative Innovation Center of IFSA (CICIFSA), Shanghai Jiao Tong University, Shanghai 200240, China \\ ${ }^{5}$ School of Physics Science and Technology, ShanghaiTech University, Shanghai 200031, China
}

A hybrid capillary discharge waveguide formed by injecting low-pressure hydrogen $(<3.8$ Torr $)$ into a pure ablative capillary is presented to supply the stable guiding for multi-GeV laser wakefield acceleration. The injected low-pressure gas only provides the seed plasma for ablative discharge breakdown, like the adsorbed gas in the inner wall of the ablative capillary. With this hybrid capillary, a stable discharge with low jitter $(\sim 5 \mathrm{~ns})$ can be achieved in a simple way, and the plasma density inside can also be controlled in a range of $\sim 0.7 \times 10^{18} \mathrm{~cm}^{-3}-1.2 \times 10^{18} \mathrm{~cm}^{-3}$ within $\sim 150 \mathrm{~ns}$ plasma channel temporal window. Furthermore, the hybrid capillary can also be easily extended to a longer length by adding multiple segments, and femtosecond laser pulses can be well guided both in the single and multiple segments mode. With these advantages, the hybrid capillary may provide an attractive plasma channel for multi-GeV-scale laser wakefield acceleration.

\section{INTRODUCTION}

Owing to their high acceleration gradients, laser wakefield accelerators ${ }^{1,2}$ (LWFAs) are considered as extremely compact high-energy electron beam sources, and great progresses have been achieved in the past few years ${ }^{3-11}$. The generation of high-energy electron beams up to $4.5 \mathrm{GeV}$ from a capillary discharge waveguide $^{7}$, as well as the two-stage coupling of independent LWFAs ${ }^{8}$, have made the LWFA even more

\footnotetext{
a)wentao.li@strath.ac.uk

b) michaeljs_liu@siom.ac.cn
} 
attractive in the production of all-optical x-ray ${ }^{12}, \gamma$-ray sources ${ }^{10,13}$, and free-electron lasers ${ }^{14}$ (FELs).

In theory, the LWFA offers great advantages with respect to conventional accelerators. However, the energy gain is limited by the laser depletion length and dephasing length ${ }^{2}$. The discharge capillary waveguide, as a plasma channel technique to guide laser pulses over long distances of several centimeters has been used in experiments to achieve a multi-GeV energy gain ${ }^{3,7,15,16}$. The plasma channel is formed inside the waveguide after discharge and keeps the laser pulse focused when the matching condition is satisfied $^{2}$. Two different varieties of discharge capillary have been used to generate such plasma channels: the ablative capillary ${ }^{17}$, which is generally made of polyethylene or acrylic, and easily obtained by mechanical processing ${ }^{15,16}$; and the gas-filled capillary ${ }^{18}$, which is made of high-melting-point materials such as sapphire ${ }^{19}$ or diamond ${ }^{20}$. These high-melting-point gas-filled capillaries need to be laser-machined and generally have a fixed length after the processing. Furthermore, it is also difficult to stick the gasfilled capillaries together without any gas leaking and deformation. Therefore, they cannot be extended up to an arbitrary length easily by sticking together. On the contrary, the ablative capillary can be made in arbitrary lengths or easily extended to segmented forms ${ }^{15,21}$ for cascaded LWFA.

Although the ablative capillary is easily available, the characteristic breakdown jitter ${ }^{22}$ of hundreds of nanoseconds that occurs during the discharge process leads to unstable discharges compared to the gasfilled capillary. Because the discharge mechanisms in both two kinds of discharge capillaries are different. In the ablative capillary, the adsorbed gas plays an important role in the discharge, thus the remarkable jitter occurred in the discharge of ablative capillary may be due to the exhaustion of the adsorbed gas in the capillary inner wall surface. The discharge mechanism ${ }^{23}$ in the ablative capillary can be attributed to the secondary electron emission avalanche (SEEA). In such a mechanism, electrons are emitted from the cathode triple junction and bombard the surface of the capillary, thus leading to desorption and ionization of the adsorbed gas. Then, some of the ions contribute to the enhancement of the electric field at the triple junction, which in turn causes an increase of the electrons emitted from the triple junction itself. The final result is desorption and ionization of more adsorbed gas. Afterwards, a regenerative process occurs and quickly leads to discharge in the capillary. Unlike the ablative capillary, the discharge in the gas-filled capillary is the result of direct ionization of the filled gas, which is easily and very quickly ${ }^{18,24}$. 
Although the discharge mechanism is different in both two cases, the plasma channel formation processes are nearly the same, which is the result of the balance between Ohmic heating and cooling of the cold wall due to thermal conduction ${ }^{18,24,25}$. What the differences are the early stages in their plasma channel formation processes. In the gas-filled capillary, the plasma temperature increases as the discharge current increase and the filled gas begin to be ionized until fully ionization. During the further evolution, the plasma temperature achieves its maximum, and in this stage the balance of Ohmic heating and thermal conduction to the cold capillary wall gives a plasma temperature profile with its maximum on axis minimum on the wall. This monotonic radially distributed plasma temperature results in a minimum plasma density on axis, which gives a plasma channel formation ${ }^{18,24}$. In the ablative capillary, once the discharge begins, due to the significant heating the inner wall of the capillary will be ablated and vapored firstly. Then as the discharge current increase, the plasma temperature also increases and the ablated and evaporated gas begin to be ionized, and the fast plasma compression occurs from the periphery to on-axis. After a further evolution, owing to the balance of Ohmic heating and thermal conduction to the cold capillary wall, a plasma channel, which a parabolic density profile with minimum on-axis, is formed to guide the laser pulse ${ }^{25}$. Therefore, although the discharge mechanisms are different, the mainly plasma channel formation processes in both two cases are nearly the same except that the ablative capillary has processes of wall ablation and evaporation and plasma compression in the early stages.

Because of the fact that discharge of the ablative capillary is triggered by the ionization of the adsorbed gas in the inner wall and propelled by the ablation and evaporation of the inner wall. Therefore, after some repeated discharge processes, the inner wall of the capillary would become smooth and the adsorbed gas would also get exhausted, so that the discharge in the ablative capillary becomes unstable.

In order to improve the discharge instability and reduce the jitter in the ablative capillary, an additional igniting laser pulse is usually used in discharge experiments. The laser pulse is typically focused coaxially $^{22}$ (the axial laser ignition (ALI) method) or transversely ${ }^{26}$ (the transverse laser ignition (TLI) method) into the capillary. In the ALI method, the focused laser is used to heat and evaporate the inner wall and the electrodes along the whole capillary. In the TLI method, a copper wire is placed in a hole that is manufactured perpendicularly to the capillary axis, and the laser is focused onto the top of the copper wire from the transverse direction to ionize the wire itself. Both methods produce the seed plasma for 
breakdown and allow a reduction of the jitter. In particular, with the ALI and TLI methods the jitter is reduced to $5 \mathrm{~ns}$ and $17 \mathrm{~ns}$, respectively. However, the ALI method enormously reduces the lifetime of the capillary, whereas the structure of the capillary in the TLI method is very complicated.

In this work, a hybrid capillary obtained by injecting a low-pressure hydrogen gas into a pure ablative capillary is proposed to supply the low-jitter capillary discharge channel for the LWFA. The filled Lowpressure hydrogen gas in the ablative capillary is used to replace the adsorbed gas layer and provide the seed plasma for the breakdown. After the trigger of the discharge, the low-ionization-threshold hydrogen will be first ionized to form the low-density seed plasma. With this kind of seed plasma filling the whole capillary, the breakdown can be further initialized, and then the inner wall of the capillary will be ablated and ionized, thus forming the plasma channel that guides the laser pulse.

This paper is organized as follows. In Sec. II, we mainly lay out the experimental setup, including the capillary discharge system and plasma density measurement system. In Sec. III, the discharge stability and discharge delay of the hybrid capillary in different gas pressures are firstly demonstrated. Then the on-axis plasma density is measured in different gas pressures. After that, the plasma channel and optical guiding experiments of one-segment and multi-segment hybrid capillaries are investigated. Finally, the conclusions are given in Sec. IV.

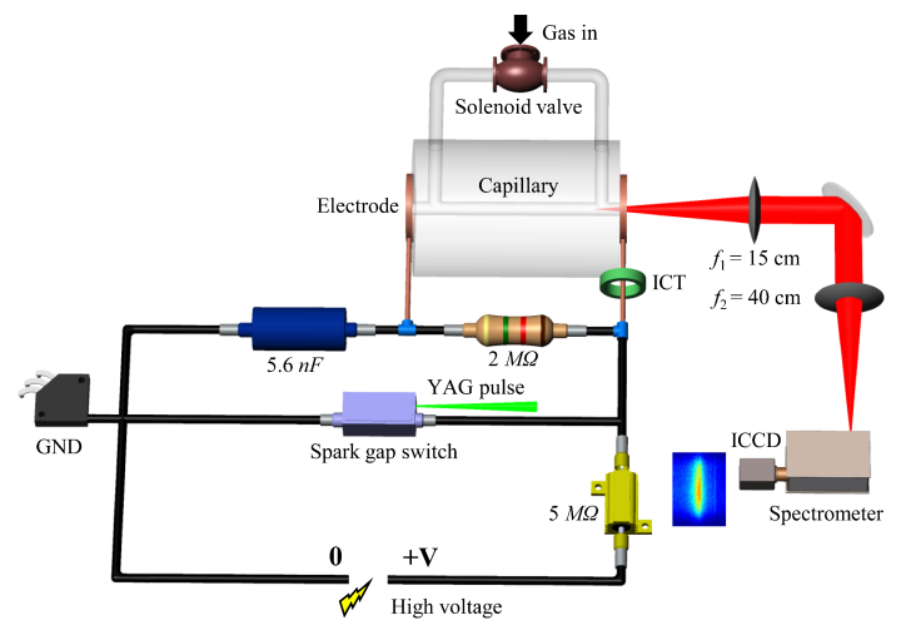

FIG. 1. Schematic diagram of the hybrid capillary and experimental setup.

\section{EXPERIMENTAL SETUP}


As shown in Fig. 1, the structure of the hybrid capillary is the same as the gas-filled capillary. Lowpressure hydrogen gas is injected into the capillary via two 500- $\mu$ m-diameter slots, which are located 1.5 $\mathrm{mm}$ away from each end of the capillary. The experimentally tested hybrid capillary was 60-mm-long with a diameter of $500 \mu \mathrm{m}$ and was made of polymethyl methacrylate (PMMA). Moreover, it could be possible to further increase the length of the capillary by adding multiple segments to a cascaded form.

The discharge circuit consisted of a $5.6 \mathrm{nF}$ capacitor charged to a voltage between $24 \mathrm{kV}$ and $30 \mathrm{kV}$. A spark gap switch, which was driven by the synchronized Nd:YAG laser pulse, was used to replace the thyratron in the discharge circuit to trigger the discharge ${ }^{27}$. The trigger pulse and the discharge current were monitored by a fast photodiode and an integrating current transformer (ICT), respectively, and the signals were recorded by a Tektronix-MDO3104 oscilloscope.

The hydrogen gas pressure inside the capillary was measured by a calibrated gas pressure transducer ${ }^{27}$ (EPIH, Measurement Specialties). During the discharge process, the total plasma density was measured by Stark broadening effect of the $\mathrm{H}_{\alpha}$ line with a spectrometer (1200 lines/mm grating) equipped with an intensified charge-coupled device (ICCD, Andor iStar 303i). The plasma emission spectra were collected longitudinally by two lenses $(f=15 \mathrm{~cm}$ and $40 \mathrm{~cm})$ and imaged onto the entrance slit (10 $\mu \mathrm{m}$ width) of the spectrometer. The width of the $\mathrm{H}_{\alpha}$ spectral line was measured by scanning the time delay between the triggering laser pulse and the gate signal of the ICCD, and the width of the gate signal was 20 ns. According to the Stark broadening effect, the plasma density can be further obtained by the formula ${ }^{28,29} n_{e}\left[\mathrm{~cm}^{-3}\right]=8.02 \times 10^{12}\left(10 \times \Delta \lambda_{\text {Stark }}[\mathrm{nm}] / \alpha\right)^{3 / 2}$, where $\alpha$ is an experimental parameter and can be chosen as ${ }^{30} 1.8 \times 10^{-2}$ for $10^{17} \mathrm{~cm}^{-3}$ and $2.2 \times 10^{-2}$ for $10^{18} \mathrm{~cm}^{-3}$. Furthermore, the Stark broadening effect can also be used to measure the transverse plasma density distribution via the distinct measurement of the broadening of the $\mathrm{H}_{\alpha}$ spectral line in the radial direction ${ }^{15,16,26,31}$.

\section{EXPERIMENTAL RESULTS AND DISCUSSION}

\section{A. Discharge stability measurement of the hybrid capillary}

In order to compare the discharge breakdown jitter and the discharge stability of the pure ablative capillary and the hybrid capillary, the discharge time delay (DTD) and the accumulated discharge current 
signals have been both investigated in 50 successive shots. In this context, the DTD represents the time difference between the triggering laser pulse and the beginning of the discharge process. In addition, during the whole discharge process, the applied voltage was kept constant at $24 \mathrm{kV}$. The results are shown in Fig. 2. As can be noted from Fig. 2(a), with no gas injection, the DTD of the pure ablative capillary remains almost constant $(\sim 125 \mathrm{~ns})$ with low jitters in the first 20 shots; then, in the next 30 shots, it becomes very unstable and is affected by a dramatical jitter. In contrast, with 3.8-Torr-gas-pressure injection, the discharge processes in the hybrid capillary appear very stable and the DTD remains constant $(\sim 123 \mathrm{~ns})$ and with low jitters for the whole duration of the 50 shots. This DTD is very close to the one of the pure ablative capillary, indicating that the discharge process in the hybrid capillary with 3.8-Torrhydrogen-gas injection is similar, but more stable, to the one in the pure ablative capillary. The physical mechanism that can possibly explain this phenomenon could be that the injected low-pressure gas in the hybrid capillary only plays the role of the adsorbed $\operatorname{gas}^{23,32}$ in the pure capillary and provides the seed plasma sources for the discharge breakdown. Once the SEEA occurs, the capillary inner wall is then ablated during the discharge process. The same result can also be obtained from the accumulated discharge-current signals in both kinds of capillaries, as shown in Figs. 2(b) and 2(c). The discharge current signals in the pure ablative capillary are very different from each other; however, they become very consistent in the hybrid capillary with 3.8-Torr-gas-pressure injection, and the jitter accounts for about 5 ns only. This low-jitter hybrid capillary is comparable with the structures in the ALI and TLI methods, but it has a longer lifetime. 

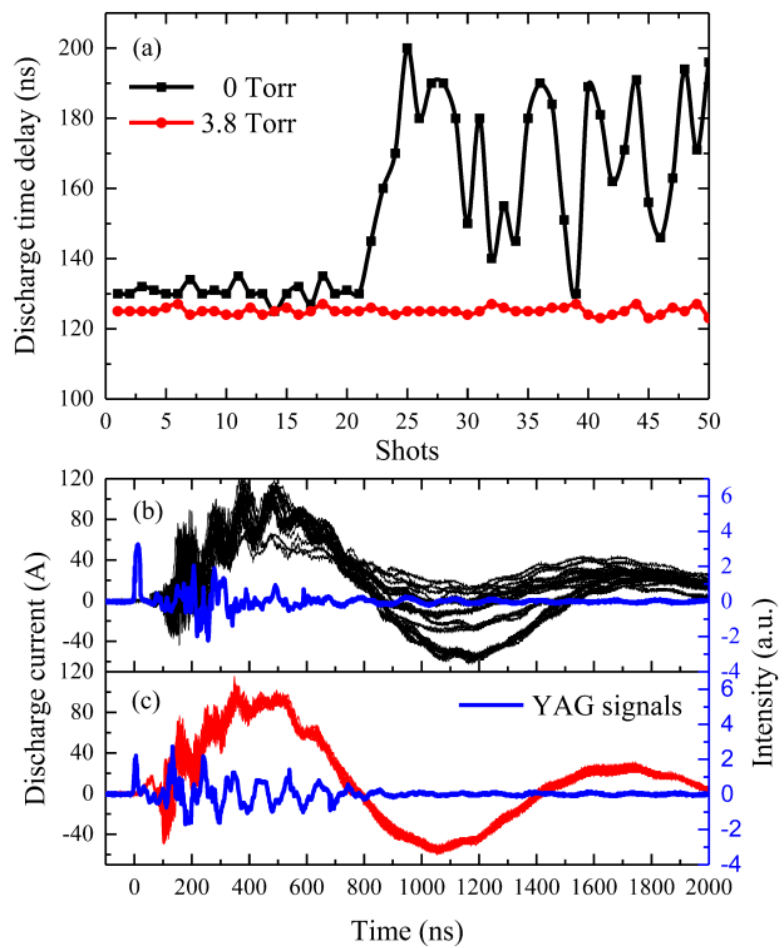

FIG. 2. DTD (a) and accumulated discharge current signals (b, c) in 50 successive shots with no gas (black curve) and low-pressure (3.8 Torr) hydrogen gas injected into the capillary (red curve). Blue curves in (b) and (c) are the YAG signals of the trigger pulse. The applied voltage is kept constant at $24 \mathrm{kV}$ during the whole discharge.

It is worth noting that with the gas injection, the jitters can be obviously decreased both in the new and in the excessively ablated capillaries. For the latter one, even if the gas is no longer injected, the jitters remain still very low for the next several shots (normally less than 3 shots). After that, the jitter would increase sharply again, returning back to the state of unstable discharge. This can be due to the fact that, though without any additional gas injection, the residual of the previously injected gas may adsorb on the inner surface of the capillary and contribute to the stability in the first several shots. Once the residual injected gas gets exhausted during the discharge process, the capillary with no gas-injection would return to the excessively ablated state.

Similar to the case in the ALI and TLI methods, the increase of the voltage applied to the capillary can shorten the DTD. For example, in the presence of the gas at a pressure of 3.8 Torr, the DTD decreases from $\sim 123 \mathrm{~ns}$ to $\sim 100 \mathrm{~ns}$ if a voltage of $28 \mathrm{kV}$ is applied. Furthermore, the DTD of the hybrid capillary is 
also influenced by the injected gas pressure. Fig. 3 shows the 50-shots averaged DTD, and the corresponding jitters, at different gas pressure when the applied voltage is $24 \mathrm{kV}$. It can be observed that, though low jitters are always achieved at different gas pressures, the DTD gets shortened as the gas pressure inside the capillary increases. This is explained from the fact that as the gas pressure increases, more seed plasmas are provided, and the regenerative process of the SEEA is further accelerated, leading to the shortening of the time delay. However, with the injection of higher-pressure gas, the hybrid capillary may tend to work as a gas-filled capillary, thus showing a further shortened DTD.

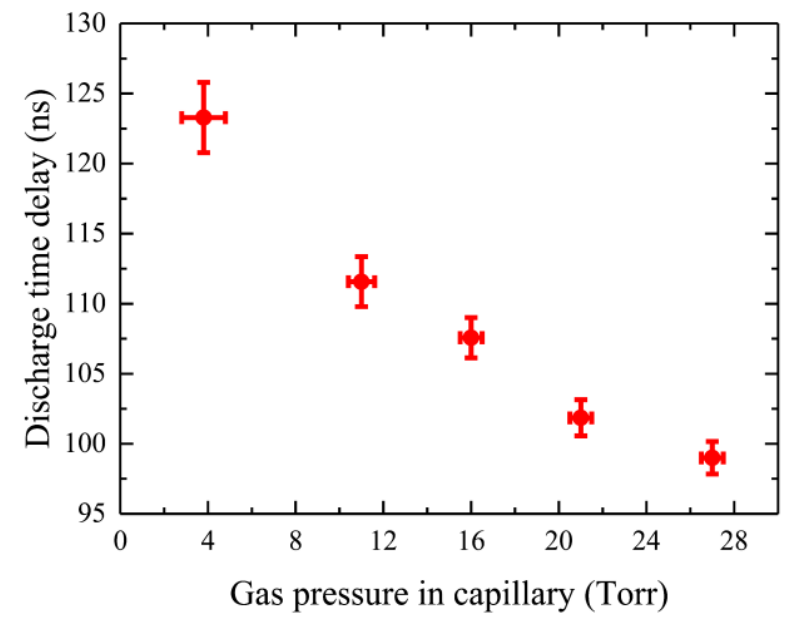

FIG. 3. 50-shots averaged DTD, and corresponding jitters, at different gas pressures in the case of 24-kV applied voltage.

\section{B. On-axis plasma density measurement}

Unlike the gas-filled or pure ablative capillary, the total plasma (TP) inside the hybrid capillary consists of two portions: the gas plasma (GP) and the ablative plasma (AP), which are produced by the ionization of the injected gas and the ablation of the inner wall, respectively. In order to quantitatively analyze the ratio of each source, TP and GP densities were measured in the hybrid capillary by means of a spectrometer and a calibrated gas pressure transducer, respectively. By measuring the gas pressure in the capillary with the transducer, the fully ionized GP density can be obtained by the ideal gas $1 \mathrm{law}^{27}$. For the sake of description, all the plasma densities mentioned in this subsection are on-axis plasma densities. Fig. 4 shows the temporal evolution of the measured TP density in the hybrid capillary with (red and blue dots) and without (violet dots) gas-injection. The applied voltage is $24 \mathrm{kV}$ in both cases, and the temporal 
evolution of the discharge current follows the black curve in Fig. 4. Each point in the figure represents the average density of four shots under the same condition. It can be found that when no gas is injected, the hybrid capillary works as a pure ablated capillary and the TP density just equals the AP density, as shown by the violet curve. Due to the continuous ablation and ionization of the inner wall during the discharge process, the AP density increases from zero to about $2.0 \times 10^{18} \mathrm{~cm}^{-3}$ at the end of the first discharge oscillating period.

Once the gas is filled into the hybrid capillary, the TP density is comprised of the GP density and the AP density. Fig. 4 also shows the temporal evolutions of the TP density in the cases in which the gas pressure (fully ionized GP density) inside the capillary is 3.8 Torr $\left(2.36 \times 10^{17} \mathrm{~cm}^{-3}\right)$ and 21.0 Torr $\left(1.32 \times 10^{18} \mathrm{~cm}^{-3}\right)$; the former and the latter cases are indicated by red dots and blue squares, respectively. For the sake of comparison, the "ideal" TP density in both cases, calculated by the sum of the measured AP density (without gas injection) and the fully ionized GP density, was also showed in Fig. 4 in blue and red curves, respectively. It can be found that, when the gas pressure inside the capillary is 3.8 Torr, the measured TP density (red dots) increases along the AP density curve (without gas injection) in the range of 120-300 ns. However, as the discharge current increases, the measured TP density stays higher than the AP density and well consistent with the "ideal" TP density curve (red curve) in the range of $\sim 400-550$ ns, which is also around the peak of the discharge current. This means that, in the case of a gas pressure of 3.8 Torr, the injected GP only takes a small portion of the measured TP and the major part is provided by the ablation of the capillary. In addition, the AP density in the hybrid capillary filled with low-pressure gas is almost the same as in the absence of gas. This behavior indicates that the low-pressure gas injected in the hybrid capillary only replaces the adsorbed gas in the inner wall surface, and the discharge process is still the same as in the pure ablative capillary. 


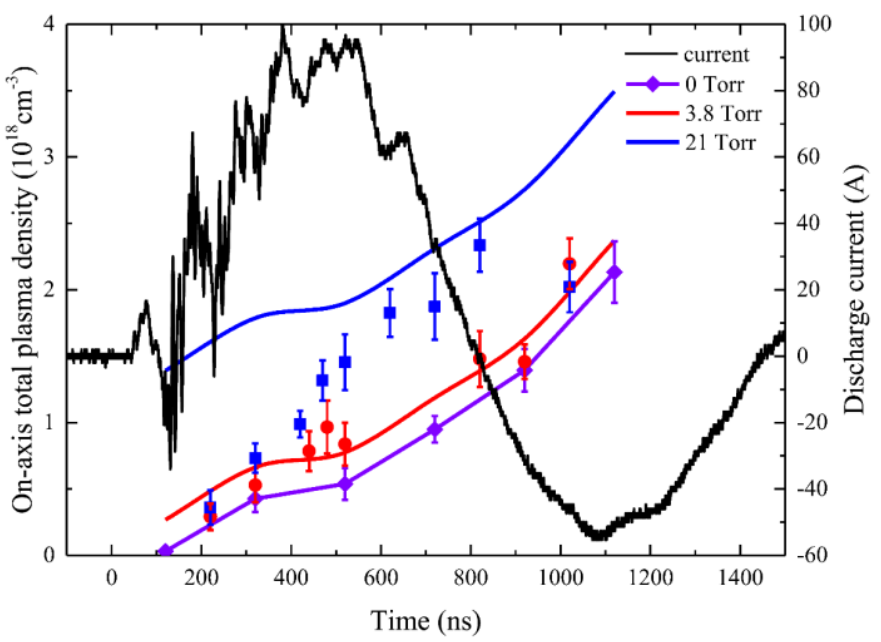

FIG. 4. Evolution of the measured (dots) and calculated "ideal" (curves) on-axis TP densities in the hybrid capillary, in the cases of no gas injection (violet), 3.8-Torr-pressure gas injection (red), and 21.0-Torrpressure gas injection (blue).

However, when the gas pressure inside the hybrid capillary is increased to 21.0 Torr, the evolution of the measured TP density (blue squares) becomes more complicated, as shown in Fig. 4. It is worth to note that, because the discharge current profile is mainly related to the applied voltage, so that the discharge current shape in different gas pressures under the same applied voltage is similar to each other except for the discharge delays, as shown in Fig. 3. Therefore, in order to share one discharge current in Fig. 4, we have shifted the data points in the case of 21.0 Torr in postprocess according to the discharge delay differences $(\sim 20 \mathrm{~ns})$. It can be observed that the measured on-axis TP density increases along the AP density curve in the beginning, whereas it gradually gets closer to the "ideal" TP density curve (blue curve) as the discharge current increases. This is because more hydrogen molecules are ionized as the discharge current increases; thus, the ionization produced GP density is comparable with the AP density in the pure capillary. With the time delay becomes about $820 \mathrm{~ns}$, the GP density achieves its maximum and the measured TP density reaches its peak value of $2.34 \times 10^{18} \mathrm{~cm}^{-3}$. After the peak, due to the recombination of the hydrogen ions, the measured TP density begins to decrease and goes back to the AP density again. This evolution of the measured on-axis TP density is similar to the one observed in the gas-filled capillary $^{33,34}$. Unlike the low-pressure-gas case (3.8 Torr), in the high-pressure-gas case (21.0 Torr) the GP density is comparable with the AP one. Furthermore, the measured TP density is generally lower than the 
"ideal" TP density in the case of high-pressure gas, which indicates that the ablation in the hybrid capillary is mitigated to a certain extent, and the hybrid capillary tends to work as a gas-filled capillary.

In the hybrid capillary, the lower limit of the TP density is the AP density, which is determined by the discharge current and the internal diameter of the capillary. For practical applications requiring a low plasma density, such as the multi-GeV laser wakefield electron acceleration, a low-pressure gas $(<3.8$ Torr) can be injected into the hybrid capillary to stabilize the discharge process, while maintaining the AP density low $\left(<2.0 \times 10^{18} \mathrm{~cm}^{-3}\right)$. As specified above, by adjusting the injected gas pressure in the hybrid capillary, the plasma density can be easily controlled, given the more flexibility of the hybrid capillary with respect to the pure ablative one.

\section{Plasma channel and optical guiding investigation}

Moreover, in order to investigate the plasma channel formation and optical guiding in the hybrid capillary, the transmittance of a guiding laser pulse passing through the capillary and the transverse total plasma density were measured. A laser pulse with duration of $45 \mathrm{fs}$, wavelength of $800 \mathrm{~nm}$, and energy of $10 \mathrm{~mJ}$ was focused at the entrance of the hybrid capillary with a spot size of $45 \mu \mathrm{m}$ (radius at which the intensity drops to $1 / \mathrm{e}^{2}$ of the peak value). A CCD camera was used to measure the energy and the spatial mode of the transmitted laser pulse at the exit of the capillary by an imaging system. By controlling the time delay between the triggering pulse and the femtosecond laser pulse, the temporal evolution of the laser transmittance during the discharge process was measured for the hybrid capillary (with 3.8 Torr gasinjection) and the gas-filled capillary (with 21.0 Torr gas-injection). The results obtained are shown in Fig. 5(a). The laser transmittance here is defined as the ratio of the total intensity of a certain area $(\sim 150 \mu \mathrm{m})$ around the center at the exit of the capillary to the whole input focal spot intensity ${ }^{35}$. Similar to Fig. 4 , the data points in 21.0 Torr case have also been shifted in the postprocess. It can be found that, in the case of the hybrid capillary (red curve), the transmittance is below $10 \%$ at the beginning of the discharge, whereas it increases dramatically to above $90 \%$ as the discharge current achieves its peak. Moreover, the temporal window with a transmittance greater than $90 \%$ is $\sim 580-730$ ns. After the temporal window, the transmittance drops to below 10\% again. In addition, the spatial mode of the transmitted laser was also detected in the case of hybrid capillary (with 3.8 Torr gas-injection), as shown in Fig. 5(c-h). It can be seen that, when there is no discharge, as shown in Fig. 5(d), the transmitted laser energy is mainly 
distributed around the periphery and nearly without energy gathered in center, which lead to a low transmission (below 10\%). When the discharge begins, as time goes on, the transmitted laser energy gathers slowly toward the center and after the temporal window, the transmitted laser energy begins to diffuse around again. Furthermore, in order to measure the matched spot size of the transmitted laser more accurately in the case of hybrid capillary, the transverse TP density distribution was also measured by means of the distinct measurement of the broadening of $\mathrm{H}_{\alpha}$ spectral line in the radial direction, and the results is shown in Fig. 5(b). It can be found that the parabolic plasma density distribution profile is formed during time delay of $\sim 500-800 \mathrm{~ns}$, which is agreement with the results of the laser transmission measurement and optical guiding measurement. In Fig. 5(b), by fitting the transverse plasma density profile at $\sim 700 \mathrm{~ns}$ (blue curve), a matched transmitted laser spot size can also be obtained to be about 65 $\mu \mathrm{m}$, which is almost agreement with the optical guiding measurement result and the formula calculation $^{24,25} w_{m}[\mu \mathrm{m}] \approx 1.48 \times 10^{5} \sqrt{R[\mu \mathrm{m}]} /\left(n_{e 0}\left[\mathrm{~cm}^{-3}\right]\right)^{1 / 4}$, where $R$ is radius of the capillary and $n_{e 0} \approx 1.13 \times 10^{18} \mathrm{~cm}^{-3}$ is the on-axis TP density. Moreover, because of the high laser transmission rate in the time delay range of $\sim 580-730 \mathrm{~ns}$, so by controlling the incoming time of the driven laser pulse during this plasma channel temporal window, a controllable plasma density range can be obtained in LWFA experiments. As shown in Fig. 4, this controllable plasma density range is $\sim 0.7 \times 10^{18} \mathrm{~cm}^{-3}-1.2 \times$ $10^{18} \mathrm{~cm}^{-3}$, and by investigating the Stark broadening effect in radial direction, the corresponding matched spot size range is indicated to be $\sim 63-68 \mu \mathrm{m}$. Therefore, by investigating the plasma channel formation and optical guiding in the case of the hybrid capillary, the plasma channel temporal window is measured to be $\sim 580-730 \mathrm{~ns}$ and the corresponding matched spot size range is $\sim 63-68 \mu \mathrm{m}$.

For the case of gas-filled capillary, as shown in Fig. 5(a) by blue curve, the temporal evolution of the laser transmittance is similar to the hybrid one, except that the peak value is achieved about 180 ns earlier than the latter. This time difference may be attributed to the discharge time delay difference of both two cases and the differences of early stages in their plasma channel formation processes. This result is consistent with previous experiments ${ }^{20,36}$. By combing the on-axis total plasma density in Fig. 4 (blue curve) and the matching formula above, the matched spot size in the case of gas-filled capillary is indicated to be $\sim 67 \mu \mathrm{m}$, where the on-axis TP density is $\sim 1.5 \times 10^{18} \mathrm{~cm}^{-3}(\sim 500 \mathrm{~ns})$. Therefore, all the results indicate that the plasma channels in both cases can be developed during the temporal window, which is 
long enough for laser guidance.
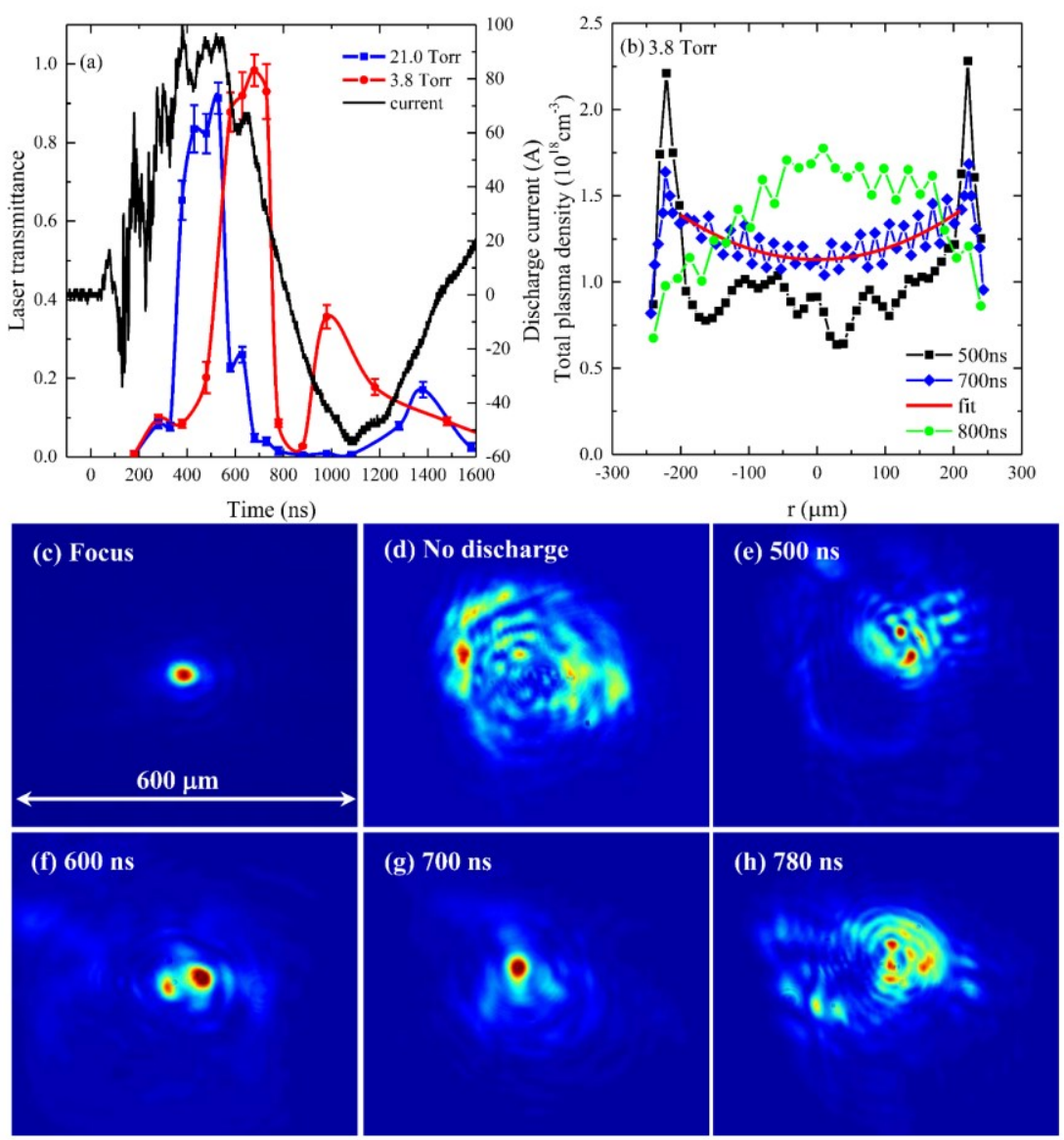

FIG. 5. Temporal evolution measurement (a) of the laser transmittance, plasma channel evolution measurement (b) and optical guiding evolution measurement (c-h). The gas pressure inside the capillary in (b) and (c-h) is 3.8 Torr. By measuring the transverse total plasma density, the macheted spot size at $\sim 700$ ns time delay was obtained to be $\sim 65 \mu \mathrm{m}$.

\section{Plasma channel investigation of two-segment capillary}

Compared to the gas-filled capillary made of sapphire or diamond, the hybrid capillary can be easily extended to a longer length by adding multiple segments, as shown in Fig. 6(a), where the structure of a two-segment $(30 \mathrm{~mm}+30 \mathrm{~mm}$ ) hybrid capillary tested in our experiments is schematically represented. The gas was injected into the capillary via the slots located $1.5 \mathrm{~mm}$ away from the ends of each segment and the gas pressures inside each segment can be controlled independently. With this combination, the length of the modular hybrid capillary is the same as the one of the single-segment capillary tested above. 
In order to consistently investigate the laser transmittance in the modular capillary and compare it with that in the single-segment capillary, the injected gas pressure in both cases was the same. Fig. 6(b) shows the transmittance evolutions of the laser passing through the modular capillary in the cases of 3.8 Torr gasinjection (red curve) and 21.0 Torr gas-injection (blue curve). Similarly, the data points in Fig. 6 (a) in 21.0 Torr case have also been shifted in the postprocess. By comparing the results in Fig. 6(b) with those in Fig. 5(a), it can be found that the laser transmittance curves in the modular capillary are quite similar to the ones in the single-segment capillary, both in low-pressure and high-pressure cases. This result demonstrates the feasibility of the modular hybrid capillary and the consistency with the single-segment one. Therefore, the hybrid capillary can be extended to multiple segments easily without changing the plasma density distribution and plasma channel formation.

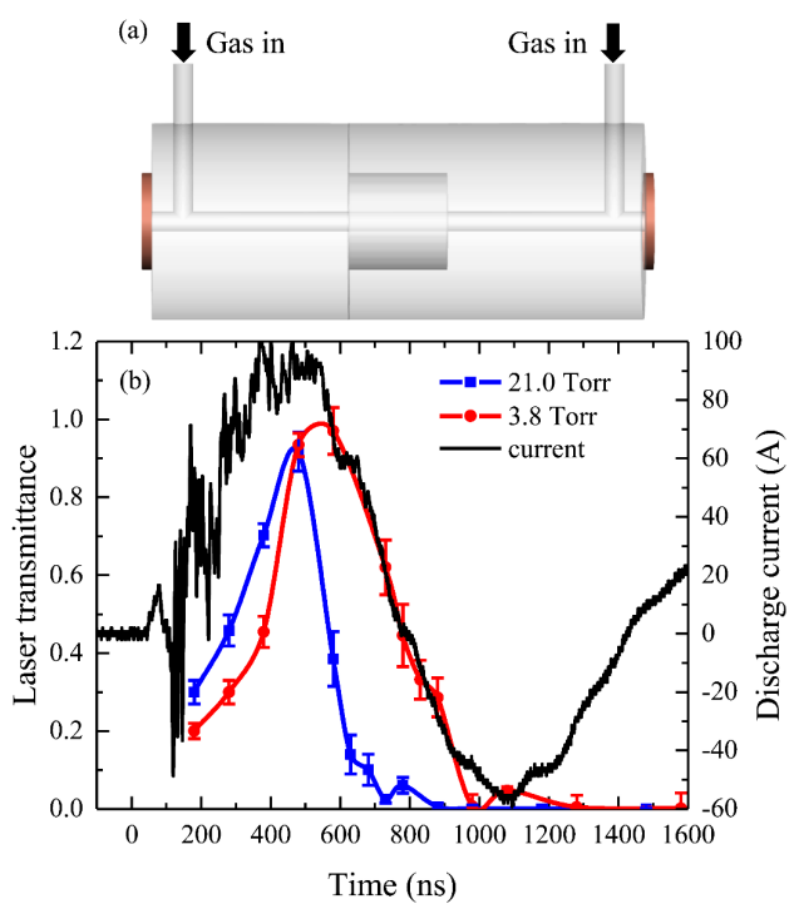

FIG. 6. Structure of the modular capillary comprised of two segments tested in experiments (a) and transmittance evolution of the (b) laser passing through the modular capillary during the discharge process (black curve), in the cases of gas injection at 3.8 Torr (blue curve) and 21.0 Torr (red curve).

\section{CONCLUSIONS}

In conclusion, we have proposed a hybrid capillary, which is formed by injecting low-pressure hydrogen 
$(<3.8$ Torr) into a pure ablative capillary. By investigating the DTD and discharge current signals of the hybrid and pure ablated capillaries, it can be found that the DTD ( $\sim 23 \mathrm{~ns}$ with $\sim 5 \mathrm{~ns}$ jitters $)$ and discharge processes in the hybrid capillary with 3.8 Torr gas-injection are more stable than in ablative capillaries. In order to analyze the ratio of the GP density and the AP density in the hybrid capillary in the cases of lowpressure (3.8 Torr) and high-pressure (21.0 Torr) gas injection, a spectrometer and a calibrated gas transducer have been used to measure the TP and GP densities, respectively. The results show that, in the case of low-pressure gas injection, the measured TP density is mainly provided by the inner wall ablation of the capillary, and the AP density in this case is almost equal to that in the pure ablative capillary under the same applied voltage. This indicates that the injected low-pressure gas in the hybrid capillary only replaces the adsorbed gas in the pure ablative capillary; thus, the discharge process does not change. However, in the high-pressure gas injection case, the hybrid capillary tends to behave as a gas-filled capillary. Furthermore, in order to investigate the channel formation and matched spot size in the capillary, the laser transmittance evolutions in the cases of the hybrid capillary (at low pressure) and gas-filled capillary (at high pressure) are measured. The results indicate that, the channels in both cases can be developed during temporal windows ( 150 ns duration), which is long enough for laser guidance. Moreover, during the plasma channel temporal window, by measuring the spectral broadening of $\mathrm{H}_{\alpha}$ spectral line in the radial direction, the controllable plasma density range and the corresponding matched spot size range are investigated to be $\sim 0.7 \times 10^{18} \mathrm{~cm}^{-3}-1.2 \times 10^{18} \mathrm{~cm}^{-3}$ and $\sim 63-68 \mu \mathrm{m}$, respectively. This controllable plasma density range is very promising for laser wakefield acceleration. Besides, laser transmittance curves for a two-segment capillary are also measured for different pressures of the gasinjection, and the results are similar to the ones obtained in the single-segment capillary. Therefore, the hybrid capillary combines the advantages of the pure ablative capillary and the gas-filled capillary. Not only the hybrid capillary has a stable-discharge process and a long lifetime, but it can also be extended to a longer length (such as 10-cm-scale long) by adding multiple segments. All these characteristics make the hybrid capillary an attractive proposal for the GeV-scale, or even for multi-GeV-scale laser wakefield acceleration.

\section{ACKNOWLEDGMENTS}

This work was supported by the National Natural Science Foundation of China (Grant Nos. 11425418, 
11127901, 61521093, and 11505263), the Strategic Priority Research Program (B) (Grant No. XDB16), the Shanghai Sailing Program (Grant No. 17YF1421100), the Youth Innovation Promotion Association CAS, and the State Key Laboratory Program of Chinese Ministry of Science and Technology.

\section{Reference}

T. Tajima and J. M. Dawson, Phys. Rev. Lett. 43 (4), 267 (1979).

E. Esarey, C. B. Schroeder, and W. P. Leemans, Rev. Mod. Phys. 81 (3), 1229 (2009).

W. P. Leemans, B. Nagler, A. J. Gonsalves, Cs Toth, K. Nakamura, C. G. R. Geddes, E. Esarey, C. B. Schroeder, and S. M. Hooker, Nat Phys 2 (10), 696 (2006).

J. S. Liu, C. Q. Xia, W. T. Wang, H. Y. Lu, Ch Wang, A. H. Deng, W. T. Li, H. Zhang, X. Y. Liang, Y. X. Leng, X. M. Lu, C. Wang, J. Z. Wang, K. Nakajima, R. X. Li, and Z. Z. Xu, Phys. Rev. Lett. 107 (3), 035001 (2011).

H. T. Kim, K. H. Pae, H. J. Cha, I. J. Kim, T. J. Yu, J. H. Sung, S. K. Lee, T. M. Jeong, and J. Lee, Phys. Rev. Lett. 111 (16), 165002 (2013).

Wang Wentao, Li Wentao, Liu Jiansheng, Wang Cheng, Chen Qiang, Zhang Zhijun, Qi Rong, Leng Yuxin, Liang Xiaoyan, Liu Yanqi, Lu Xiaoming, Wang Cheng, Li Ruxin, and Xu Zhizhan, Appl. Phys. Lett. 103 (24), 243501 (2013).

W. P. Leemans, A. J. Gonsalves, H. S. Mao, K. Nakamura, C. Benedetti, C. B. Schroeder, C. Toth, J. Daniels, D. E. Mittelberger, S. S. Bulanov, J. L. Vay, C. G. R. Geddes, and E. Esarey, Phys. Rev. Lett. 113 (24), 5 (2014).

S. Steinke, J. van Tilborg, C. Benedetti, C. G. Geddes, C. B. Schroeder, J. Daniels, K. K. Swanson, A. J. Gonsalves, K. Nakamura, N. H. Matlis, B. H. Shaw, E. Esarey, and W. P. Leemans, Nature 530 (7589), 190 (2016).

W. T. Wang, W. T. Li, J. S. Liu, Z. J. Zhang, R. Qi, C. H. Yu, J. Q. Liu, M. Fang, Z. Y. Qin, C. Wang, Y. Xu, F. X. Wu, Y. X. Leng, R. X. Li, and Z. Z. Xu, Phys. Rev. Lett. 117 (12), 5 (2016).

Changhai Yu, Rong Qi, Wentao Wang, Jiansheng Liu, Wentao Li, Cheng Wang, Zhijun Zhang, Jiaqi Liu, Zhiyong Qin, Ming Fang, Ke Feng, Ying Wu, Ye Tian, Yi Xu, Fenxiang Wu, Yuxin Leng, Xiufeng Weng, Jihu Wang, Fuli Wei, Yicheng Yi, Zhaohui Song, Ruxin Li, and Zhizhan Xu, Scientific Reports 6, 29518 (2016).

Zhiyong Qin, Changhai Yu, Wentao Wang, Jiansheng Liu, Wentao Li, Rong Qi, Zhijun Zhang, Jiaqi Liu, Ming Fang, Ke Feng, Ying Wu, Lintong Ke, Yu Chen, Yi Xu, Yuxin Leng, Cheng Wang, Ruxin Li, and Zhizhan Xu, Physics of Plasmas 25 (2), 023106 (2018).

S. Corde, K. Ta Phuoc, G. Lambert, R. Fitour, V. Malka, A. Rousse, A. Beck, and E. Lefebvre, Rev. Mod. Phys. 85 (1), 1 (2013).

K. Ta Phuoc, S. Corde, C. Thaury, V. Malka, A. Tafzi, J. P. Goddet, R. C. Shah, S. Sebban, and A. Rousse, Nat. Photonics 6 (5), 308 (2012).

Zhirong Huang, Yuantao Ding, and Carl B. Schroeder, Phys. Rev. Lett. 109 (20), 204801 (2012).

H. Y. Lu, M. W. Liu, W. T. Wang, C. Wang, J. S. Liu, A. H. Deng, J. C. Xu, C. Q. Xia, W. T. Li, H. Zhang, X. M. Lu, C. Wang, J. Z. Wang, X. Y. Liang, Y. X. Leng, B. F. Shen, K. Nakajima, R. X. Li, and Z. Z. Xu, Appl. Phys. Lett. 99 (9), 3 (2011).

Aihua Deng, Mingwei Liu, Jiansheng Liu, Xiaoming Lu, Changquan Xia, Jiancai Xu, Cheng Wang, Baifei Shen, Ruxin Li, Zhizhan Xu, and K. Nakajima, Plasma Science and Technology 13 (3), 362 (2011).

D. Kaganovich, P. V. Sasorov, Y. Ehrlich, C. Cohen, and A. Zigler, Appl. Phys. Lett. 71 (20), 2925 (1997).

D. J. Spence and S. M. Hooker, Phys. Rev. E 63 (1), 4 (2001).

N. H. Matlis, A. J. Gonsalves, S. Steinke, J. van Tilborg, E. H. Matlis, B. Shaw, D. E. Mittelberger, C. G. R. Geddes, and W. P. Leemans, Journal of Applied Physics 118 (20), 204506 (2015).

A. J. Gonsalves, F. Liu, N. A. Bobrova, P. V. Sasorov, C. Pieronek, J. Daniels, S. Antipov, J. E. Butler, S. S. Bulanov, W. L. 
Waldron, D. E. Mittelberger, and W. P. Leemans, Journal of Applied Physics 119 (3), 033302 (2016).

M. Levin, A. Pukhov, A. Zigler, K. Sugiyama, K. Nakajima, R. F. Hubbard, A. Ting, D. F. Gordon, P. Sprangle, and D. Kaganovich, Physics of Plasmas 13 (8), 083108 (2006).

B. Greenberg, M. Levin, A. Pukhov, and A. Zigler, Applied Physics Letters 83 (14), 2961 (2003).

H. C. Miller, leee Transactions on Electrical Insulation 28 (4), 512 (1993).

N. A. Bobrova, A. A. Esaulov, J. I. Sakai, P. V. Sasorov, D. J. Spence, A. Butler, S. M. Hooker, and S. V. Bulanov, Phys. Rev. E 65 (1), 016407 (2001).

T. Kameshima, H. Kotaki, M. Kando, I. Daito, K. Kawase, Y. Fukuda, L. M. Chen, T. Homma, S. Kondo, T. Zh Esirkepov, N. A. Bobrova, P. V. Sasorov, and S. V. Bulanov, Physics of Plasmas 16 (9), 093101 (2009).

M. W. Liu, A. H. Deng, J. S. Liu, R. X. Li, J. C. Xu, C. Q. Xia, C. Wang, B. F. Shen, Z. Z. Xu, and K. Nakajima, Rev. Sci. Instrum. 81 (3), 3 (2010).

Zhiyong Qin, Wentao Li, Jiansheng Liu, Jiaqi Liu, Changhai Yu, Wentao Wang, Rong Qi, Zhijun Zhang, Ming Fang, Ke Feng, Ying Wu, Lintong Ke, Yu Chen, Cheng Wang, Ruxin Li, and Zhizhan Xu, Physics of Plasmas 25 (4), 043117 (2018). J. Ashkenazy, R. Kipper, and M. Caner, Physical Review A 43 (10), 5568 (1991).

Vincent Detalle, René Héon, Mohamad Sabsabi, and Louis St-Onge, Spectrochimica Acta Part B: Atomic Spectroscopy 56 (6), 1011 (2001).

S. A. Flih, E. Oks, and Y. Vitel, Journal of Physics B: Atomic, Molecular and Optical Physics 36 (2), 283 (2003).

Kameshima Takashi, Hong Wei, Sugiyama Kiyohiro, Wen Xianlun, Wu Yuchi, Tang Chuanming, Zhu Qihua, Gu Yuqiu, Zhang Baohan, Peng Hansheng, Kurokawa Shin-ichi, Chen Liming, Tajima Toshiki, Kumita Tetsuro, and Nakajima Kazuhisa, Applied Physics Express 1 (6), 066001 (2008).

R. A. Anderson and J. P. Brainard, Journal of Applied Physics 51 (3), 1414 (1980).

D. G. Jang, M. S. Kim, I. H. Nam, H. S. Uhm, and H. Suk, Appl. Phys. Lett. 99 (14), 141502 (2011).

M. S. Kim, D. G. Jang, T. H. Lee, I. H. Nam, I. W. Lee, and H. Suk, Appl. Phys. Lett. 102 (20), 204103 (2013).

N. Lemos, L. Cardoso, J. Geada, G. Figueira, F. Albert, and J. M. Dias, Scientific Reports 8 (1), 3165 (2018).

C. McGuffey, M. Levin, T. Matsuoka, V. Chvykov, G. Kalintchenko, P. Rousseau, V. Yanovsky, A. Zigler, A. Maksimchuk, and K. Krushelnick, Physics of Plasmas 16 (11), 113105 (2009). 